\title{
Domiciliary self-measurement in rheumatoid arthritis and the demonstration of circadian rhythmicity
}

\author{
I. C. KOWANKO, M. S. KNAPP, R. POWNALL, AND A. J. SW ANNELL \\ From the Chronotherapeutics Research Group and the Department of Rheumatology, City Hospital, \\ Nottingham
}

SUMMARY Symptoms and signs of rheumatoid arthritis vary within the day and from day to day. Interesting and possibly important observations can be missed when evaluations are based only on outpatient measurements, which are likely to be made at only one time and at infrequent intervals. We have found that patients can measure their own grip strength and finger joint sizes at home, and simultaneously assess overall pain and stiffness on numerical scales. Measurements made by patients were reproducible when made at the same time of day if on the same treatment. The patient's subjective assessment of pain and stiffness is a useful measure of the severity of rheumatoid arthritis. These pain and stiffness ratings were found to be well correlated with the patient's objective measurements of finger joint size and grip strength. Information not otherwise available can be collected by studying patients at home with these self-measurement techniques. These have allowed the demonstration of circadian variations in the signs and symptoms of rheumatoid arthritis and improved the evaluation of drugs studied in clinical trials.

The patient's subjective assessment of pain, pain relief, and stiffness are used by doctors during clinical work and by those conducting clinical trials. ${ }^{1-3}$ Patient questionnaires have often been issued and found helpful. ${ }^{4}$ Pain and relief of pain are important to patients, and their opinion on this may be the most meaningful index of drug effectiveness. ${ }^{5}$ However, it is also useful to have objective measurements of signs and symptoms. In rheumatoid arthritis grip strength $^{6-10}$ and finger joint $\operatorname{sizes}^{811-13}$ have been measured and are traditionally made by clinical investigators or doctors. We teach the patients to measure their own grip strength and finger joint sizes and instruct them to do this at home. This type of domiciliary approach was earlier used by us in studies of renal failure ${ }^{14}$ and hypertension. ${ }^{16}$ Patients measure their signs and symptoms frequently at home, allowing studies of circadian variations. Serial measurements made over longer periods can be used to follow the course of the disease and the influence of treatment.

It is important to know if a patient's measurements are reproducible and whether objective measurements are correlated with subjective assessments. These domiciliary methods have a potential to

Accepted for publication 10 September 1981.

Correspondence to Dr I. C. Kowanko, City Hospital, Hucknall Road, Nottingham NG5 1PB. assist in research and to allow better clinical monitoring of a patient's progress. This approach has now been successfully tried in the study of several conditions and has been used by our patients with arthritis.

\section{Patients and methods}

The patients were those taking part in a trial of the therapeutic effectiveness of flurbiprofen contrasting different times of day and frequencies of administration. The design of the trial and its results have been published elsewhere. ${ }^{1718}$ With the information collected differences were demonstrated in the effectiveness of the drug attributable to the time of day of treatment, and twice daily dosing was shown to be superior to 4 times daily dosing. All patients measured their signs and symptoms at 6 fixed times of day for the 3 days at the end of each of 4 treatment periods, that is, on 72 occasions altogether.

Patients were given verbal and written instructions on the use of equipment, and practised under supervision. During the trial results were recorded on diary cards. Each patient used the same equipment throughout the study.

Grip strength was measured with an inflatable grip bag attached to an aneroid sphygmomanometer. The bag was inflated to an initial pressure of $20 \mathrm{mmHg}$. With the arm resting on a table the bag was squeezed 
as hard as possible. Patients recorded the highest reading of 3 attempts for each hand.

The size of proximal interphalangeal joints of the fingers and the distal interphalangeal joints of the thumbs were measured and recorded. Joint size was measured with a gauge consisting of a flexible Teflon loop attached to a spring-loaded pointer moving on a graduated scale. The loop was positioned over the thickest part of each joint and the circumference read off the scale. The sum of 10 finger joints was used in the analyses of results.

Patients assessed their overall stiffness and pain subjectively and marked horizontal integer scales, numbered from 0 (no pain, stiffness) to 10 (worst possible pain, stiffness).

The data collected by the patients were tested for reproducibility by analysis of variance. Nonparametric correlation coefficients between the objective and subjective assessments were also calculated.

\section{Results}

The patients measured their signs and symptoms at set times of day: $0800,1100,1300,1500,2000$, and $2300 \mathrm{~h}$. An example of a patient's measurements made over 3 days is shown in Fig. 1.

\section{SIGNS AND SYMPTOMS MEASURED OVER 3 DAYS IN A PATIENT WITH RHEUMATOID ARTHRITIS}
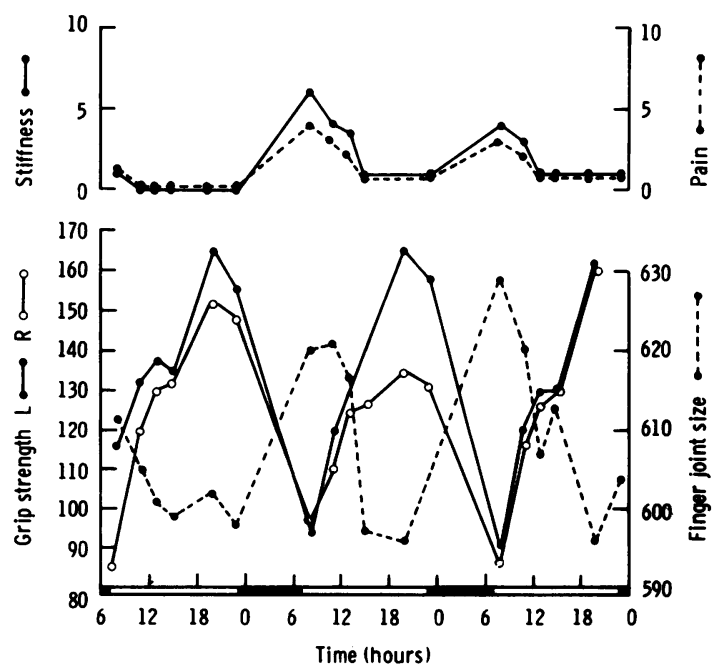

Fig. 1 Measurements of signs and symptoms were made by a patient with rheumatoid arthritis at home. The measurements are repeatable when made at the same time of day. Joint size, pain, and stiffness are greatest in the morning when grip strength is lowest, and circadian rhythmicity in these was demonstrated.
The data were amenable to analysis of variance. There was no significant variation between measurements made by the same patient at the same time from day to day during the same treatment regimen, demonstrating that patients' measurements of their grip strength and joint size are reproducible. The significant variations observed between measurements made at different times of day are indicative of circadian variations in the symptoms of rheumatoid arthritis. ${ }^{18}$ Grip strength was minimal and joint size maximal in the early hours of the morning on all treatments. Variability between patients and between treatment regimens was highly significant.

The correlation coefficients between the subjective and objective measurements were calculated. Spearman's rank correlation coefficient was calculated for all the data collected by each patient. Correlations between subjective pain and joint size, pain and grip strength, stiffness and joint size, and stiffness and grip strength were determined. The negative correlation between grip strength and subjective pain and stiffness ratings was highly significant in over $75 \%$ of patients. Joint size was positively correlated with pain and stiffness, but there were fewer patients with statistically significant $(\mathrm{p}<0 \cdot 05)$ correlations. In 11 out of 16 patients, $69 \%$, stiffness was significantly correlated with finger joint size, and 7 out of 16 patients had significant correlation between pain and joint size.

\section{Discussion}

Patients with rheumatoid arthritis measured their own grip strength and finger joint sizes at home and assessed their overall pain and stiffness on numbered scales. Analysis of variance showed that a patient's measurements of joint size and grip strength were reproducible when made at the same time of day.

Most investigators agree that a patient's assessment of pain is a useful and sensitive index in studies of rheumatoid arthritis, ${ }^{1-4}$ but the usefulness of objective measurements has been questioned. ${ }^{2}$ Downie et al. ${ }^{19}$ compared various types of pain rating scales and found that a numbered scale was the most reliable. The correlation of these subjective assessments with the objective measurements has not often been good, ${ }^{20}$ though Ingpen ${ }^{21}$ found that subjective pain ratings were well correlated with grip strength. Grip strength is a nonspecific index, as it is influenced by muscle power and pain. ${ }^{67912}$ Finger joint size has been measured by many investigators and is said to be reliable by some ${ }^{81222}$ but not by others. ${ }^{2}$ Joint size is a useful index when there is active synovial swelling but not when there are bony deformities. ${ }^{11}{ }^{13}$ The device we used to measure joint size is similar to 
that used by Boardman and Hart, ${ }^{12}$ who found it to be superior to the use of jeweller's rings.

We found that in most patients there was a statistically significant correlation between grip strength and subjective pain and stiffness ratings. Grip strength, as measured by patients with rheumatoid arthritis, was therefore a valid measure of symptom severity, as it consistently decreased with increasing pain and stiffness.

Finger joint size is an absolute measurement, unaffected by pain or stiffness. It was positively correlated with stiffness in $69 \%$ of patients $(p<0.05)$. Scott ${ }^{9}$ suggested that tissue swelling may be the cause of morning stiffness, and decreases in finger joint swelling have been noted with anti-inflammatory treatment. ${ }^{121323}$ Indeed joint size is specifically related to inflammation. ${ }^{12}$ We found that joint size generally increased as pain and stiffness increased, but only about half of the patients had significant $(p<0.05)$ correlations between joint size and pain, and of these all but one had 5 or more inflamed finger joints. Patients with active synovial inflammation of the finger joints are therefore more suitable for self-measurement of finger joint size than those without. Significant correlation does not imply cause and effect but indicates the way in which variables change in relation to each other.

The use of this method has allowed us to describe circadian variations in grip strength and finger joint sizes. ${ }^{18}$ Grip strength was minimal and joint swelling maximal in the morning, when subjective pain and stiffness scores were highest. This aspect of the disease had received little attention from other investigators and merits further study.

Patients should more often measure their own signs and symptoms at home. We have shown that measurements made by patients are reproducible and that the objective measurements are well correlated with the more conventional subjective assessments. Data collected by patients at home are more relevant than data collected in hospital or the clinic, and patients can be studied while undertaking their normal activities in their usual environment. Much more information can be obtained with only a little extra effort initially by professional staff, making closer monitoring of the long-term progress of the disease and its treatment possible. Intensive investigations, such as studies of circadian variations, can be conducted without admission to hospital. Statistical techniques that are now being developed to consider trends and changes in trends in sequential data will require information collected in this way if they are to be used without multiple visits to clinics to collect data. Vital information could be missed by assessing patients only in hospital, and it is recommended that domiciliary self-measurement be considered for most clinical investigations and for the day-to-day care of patients.

\section{References}

${ }^{1}$ Lee P, Webb J, Anderson J, Buchanan W W. Method for assessing therapeutic potential of anti-inflammatory antirheumatic drugs in rheumatoid arthritis. $\mathrm{Br}$ Med J 1973; ii: 685-8.

${ }^{2}$ Deodhar S D, Dick W C, Hodgkinson R, Buchanan W W. Measurement of clinical response to anti-inflammatory drug therapy in rheumatoid arthritis. $Q \mathrm{~J} \mathrm{Med} \mathrm{1973;} \mathrm{42:} \mathrm{387-401.}$

${ }^{3}$ Paterson J, Watson W S, Teasdale E, et al. Assessment of rheumatoid inflammation in the knee joint. Ann Rheum Dis 1978; 37: 48-52.

4 Gumpel J M. Which anti-inflammatory drugs in rheumatoid arthritis? Br Med J 1978; ii: 256.

5 Huskisson E C. Measurement of pain. Lancet 1974; ii: 1127-31.

${ }^{6}$ Wright V. Some observations on diurnal variation of grip. Clin Sci 1959; 18: 17-23.

7 Wright V, Dowson D, Longfield M D. Joint stiffness-its characterisation and significance. Biomed Eng 1969; 4: 8-14.

${ }^{8}$ Rasmussen G G. Parameter reliability. Rheumatol Rehabil 1978; suppl: 128-131.

- Scott J T. Morning stiffness in rheumatoid arthritis. Ann Rheum Dis 1960; 19: 361-8.

${ }^{10}$ Brewer K, Guyatt A R, Scott J T. Comparing grip strength. Physiotherapy 1975; 61: 118.

" Hart F D, Clark C J M. Measurement of digital swelling in rheumatoid arthritis. Lancet 1951; i: 775.

12 Boardman P L, Hart F D. Clinical measurement of the antiinflammatory effects of salicylates in rheumatoid arthritis. $\mathrm{Br}$ Med J 1967; iv: 264-8.

${ }^{13}$ Webb J, Downie W W, Dick W C, Lee P. Evaluation of digital joint circumference measurements in rheumatoid arthritis. Scand J Rheumatol 1973; 2: 127-31.

14 Hillier P, Knapp M S. The domiciliary measurement of human physiological rhythms. J Physiol 1974; 242: 45-6.

15 Hillier $P$, Knapp M S, Cove-Smith R. Circadian variations in urine excretion in chronic renal failure. $Q J$ Med 1980; 49: 461-78.

${ }^{16}$ Knapp M S, Woolfson A M J. The effects of drug therapy on circadian variations in blood pressure, and the influence of the timing of therapy. BrJ Pharmacol 1978; 64: 476P.

${ }^{17}$ Kowanko I C, Swannell A J, Mahoney P G C, Pownall R, Knapp M S. Preliminary observations on the therapeutic effectiveness of Froben at different times of day. Br J Clin Pract 1980; suppl 9: $10-4$.

${ }^{18}$ Kowanko I C, Pownall R, Knapp M S, Swannell A J, Mahoney P G C. Circadian variations in the signs and symptoms of rheumatoid arthritis and in the therapeutic effectiveness of flurbiprofen at different times of day. Br J Clin Pharmacol 1981; 11: $477-84$.

19 Downie W W, Leatham P A, Rhind V M, Wright V. Branco J A, Anderson J A. Studies with pain rating scales. Ann Rheum Dis 1978; 37: 378-81.

${ }^{20}$ Lee P, Baxter A, Dick W C, Webb J. An assessment of grip strength measurement in rheumatoid arthritis. Scand $J$ Rheumatol 1974; 3: 17-23.

${ }^{21}$ Ingpen M L. The quantitative measurement of joint changes in rheumatoid arthritis. Ann Phys Med 1968; 9: 322-7.

${ }^{22}$ Heyman E R. Variability of proximal interphalangeal joint size measurements in normal adults. Arthritis Rheum 1974; 17: 79-84.

${ }^{23}$ Smyth C J, Velayos E E, Hlad C J. A method for measuring swelling of hands and feet. Acta Rheumatol Scand 1963; 9: 293-305. 